\title{
Synthesis, urease inhibition and antimicrobial activities of some chiral 5-aryl-4-(1-phenylpropyl)-2H-1,2,4-triazole-3(4H)-thiones
}

\author{
Monazza Serwar, ${ }^{1}$ Tashfeen Akhtar, ${ }^{1}$ Shahid Hameed, ${ }^{1 *}$ and Khalid M. Khan ${ }^{2}$ \\ ${ }^{1}$ Department of Chemistry, Quaid-I-Azam University, Islamabad-45320, Pakistan. \\ ${ }^{2}$ HEJ Research Institute of Chemistry, International centre for Chemical and Biological \\ Sciences, Karachi-75270, Pakistan \\ E-mail:shameed@qau.edu.pk
}

\begin{abstract}
The synthesis of some chiral 5-aryl-4-(1-phenylpropyl)-2H-1,2,4-triazole-3(4H)-thiones (6a-i) was carried out by cyclodehydration of 1-aroyl-4-(1-phenylpropyl)thiosemicarbazides (5a-i) obtained by the condensation of isomeric halo benzoic acid hydrazides (3a-i) and (R)-(+)-1phenylpropyl isothiocyanate (4). The hydrazides (3a-i) were synthesized from the corresponding halo benzoic acids (1a-i) via esterification. The synthesis was confirmed by spectroanalytical techniques. The compounds $\mathbf{6 d}$ and $\mathbf{6 e}$ were found to be more potent urease inhibitors than the standard thiourea, depicting the $\mathrm{IC}_{50}$ values of $7.8 \pm 0.2$ and $12.4 \pm 0.2 \mu \mathrm{M}$, respectively $\left(\mathrm{IC}_{50}\right.$ of thiourea $=21.0 \pm 0.1 \mu \mathrm{M}$ ). Compounds $\mathbf{6 c}$ and $\mathbf{6 h}$ also exhibited very good urease inhibition activity with the $\mathrm{IC}_{50}$ values of $35.9 \pm 0.7$ and $31.1 \pm 0.5 \mu \mathrm{M}$, respectively. In addition the antimicrobial activities of the synthesized compounds are also being reported.
\end{abstract}

Keywords: Chiral triazoles, chiral thiosemicarbazides, urease inhibition, antimicrobial activities

\section{Introduction}

1,2,4-Triazoles and their derivatives exhibit numerous pharmaceutical ${ }^{1-9}$ and industrial applications. $^{10,11}$ Various commercially used antifungal agents belonging to this class of compounds are chiral. ${ }^{2}$ When a chiral drug interacts with a chiral receptor site; the two enantiomers of the drug interact differently and may lead to different effects. On the basis of the fact that enzymes are stereospecific, it is a need of the time to synthesize enantiopure molecules and explore their pharmacological potential. In continuation of our previous work on biologically active chiral azoles, ${ }^{12-16}$ we now report the synthesis of some chiral 5-aryl-4-(1-phenyl)propyl1,2,4-triazole-3-thiones (6a-i) along with their urease inhibition and antimicrobial activities. 


\section{Results and Discussion}

\section{Synthesis}

The synthesis of 5-aryl-4-(1-phenylpropyl)-2H-1,2,4-triazole-3(4H)-thiones (6a-i) was carried out starting from aryl carboxylic acids (1a-i) following a multistep sequence depicted in Figure 1 .<smiles>[X]c1ccc(C(=O)O)cc1</smiles>

\begin{tabular}{|c|c|c|c|}
\hline & $x$ & & $x$ \\
\hline $\mathbf{a}$ & $2-\mathrm{Cl}$ & $\mathbf{f}$ & \\
\hline b & 3-Cl & g & $2-\mathrm{F}$ \\
\hline $\begin{array}{c}\text { c } \\
\text { d }\end{array}$ & $\begin{array}{l}4-\mathrm{Cl} \\
2-\mathrm{Br}\end{array}$ & h & $3-F$ \\
\hline & $3-\mathrm{Br}$ & $\mathbf{i}$ & 4-F \\
\hline
\end{tabular}

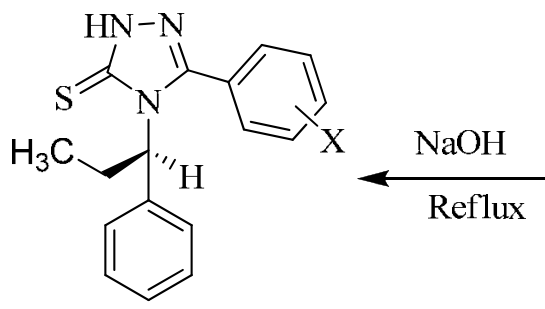<smiles>[Y]c1ccc(C(=O)NNC(=S)N[C@H](CC)c2ccccc2)cc1</smiles>

6a-i<smiles>CCN(C=S)c1ccccc1</smiles>

Figure 1. Synthesis of 5-aryl-4-(1-phenylpropyl)-2H-1,2,4-triazole-3(4H)-thiones.

The esterification of the acids was carried out using standard esterification conditions followed by hydrazinolysis to halo benzoic acid hydrazides (3a-i). ${ }^{17}$ The $(R)-(+)-1$-phenylpropyl isothiocyanate (4) was prepared from $R-(+)-1$-phenylpropan-1-amine. ${ }^{18}$ The reaction of hydrazides (3a-i) with $(R)-(+)-1$-phenylpropyl isothiocyanate resulted in the formation of chiral thiosemicarbazide derivatives (5a-i). The formation of thiosemicarbazides was indicated in the IR spectra by a shift in the carbonyl absorptions of hydrazides in the region $1662-1618 \mathrm{~cm}^{-1}$ to $1692-1665 \mathrm{~cm}^{-1}$ in thiosemicarbazides. The absorption in the region $1246-1224 \mathrm{~cm}^{-1}$ was assigned to $\mathrm{C}=\mathrm{S}$ group. The synthesis was confirmed in the ${ }^{1} \mathrm{H}-\mathrm{NMR}$ spectra by the appearance of $\mathrm{H}-1, \mathrm{H}-2$ and $\mathrm{H}-3$ proton signals in the regions of 10.29-9.63, 9.05-8.55 and 8.54-7.60 ppm, respectively. In all these compounds (5a-i), the integration curve indicated nine protons in the aromatic region confirming the introduction of the second phenyl ring in the molecule. In ${ }^{13} \mathrm{C}$ NMR spectra of compounds (5a-i), the signal for carbonyl and thiocarbonyl carbons were found in the regions of 166.7-164.2 and 183.9-183.1 ppm, respectively. The elemental analysis of thiosemicarbazides (5a-i) was in good agreement with the calculated values.

The thiosemicarbazides (5a-i) were subjected to intramolecular dehydrative cyclization in presence of $\mathrm{NaOH}$ to furnish corresponding 5-aryl-4-(1-phenylpropyl)-2H-1,2,4-triazole-3(4H)- 
thiones (6a-i). The cyclization of thiosemicarbazides (5a-i) to the products $(\mathbf{6 a - i})$ was indicated in the IR spectra by the appearance of a single absorption for $\mathrm{N}-\mathrm{H}$ and disappearance of the carbonyl absorption. The absorption band observed in the region 1514-1501 $\mathrm{cm}^{-1}$ was attributed to $\mathrm{C}=\mathrm{N}$ group. The synthesis of $(\mathbf{6 a}-\mathbf{i})$ was confirmed in ${ }^{1} \mathrm{H}-\mathrm{NMR}$ spectra by the appearance of only one relatively broad $\mathrm{NH}$ proton signal in the region of $13.01-10.55 \mathrm{ppm}$. In ${ }^{13} \mathrm{C}-\mathrm{NMR}$ spectra, the absence of the signal for carbonyl carbon and appearance of the signal for $\mathrm{C}=\mathrm{N}$ carbon in the range of 167.2-138.5 ppm also confirmed the synthesis. The elemental composition was found in good agreement with the calculated values for $\mathrm{C}, \mathrm{H}, \mathrm{N}$ and $\mathrm{S}$. The synthesis was further confirmed by the mass spectral analysis. The presence of $\mathrm{M}+2$ peaks in compounds with a chlorine or bromine atom (6a-f) confirmed the presence of these substituents.

\section{In vitro urease inhibition assay}

The compounds (6a-i) were tested for their potential to inhibit Jack bean urease and the results are tabulated in Table 1 along with the structures of the compounds for comparison purposes. Among the tested compounds, $\mathbf{6 d}$ and $\mathbf{6 e}$ were found to be more potent than the standard, with the $\mathrm{IC}_{50}$ values of $7.8 \pm 0.2$ and $12.4 \pm 0.2 \mu \mathrm{M}$, respectively (compared to standard thiourea with $\mathrm{IC}_{50}=21.0 \pm 0.1 \mu \mathrm{M}$ ). Compounds $\mathbf{6 c}$ and $\mathbf{6 h}$ also exhibited very good urease inhibition activity with the $\mathrm{IC}_{50}$ values of $35.9 \pm 0.7$ and $31.1 \pm 0.5 \mu \mathrm{M}$, respectively, whereas the activity of compounds $\mathbf{6 b}$ and $\mathbf{6 f}$ was only moderate $\left(\mathrm{IC}_{50}=75.8 \pm 1.8\right.$ and $117.7 \pm 6.8 \mu \mathrm{M}$, respectively). The compounds $6 \mathbf{a}, \mathbf{6 g}$ and $\mathbf{6 i}$ were found to be inactive at the specified concentration.

Table 1. Urease inhibition activity of the compounds 6a-i

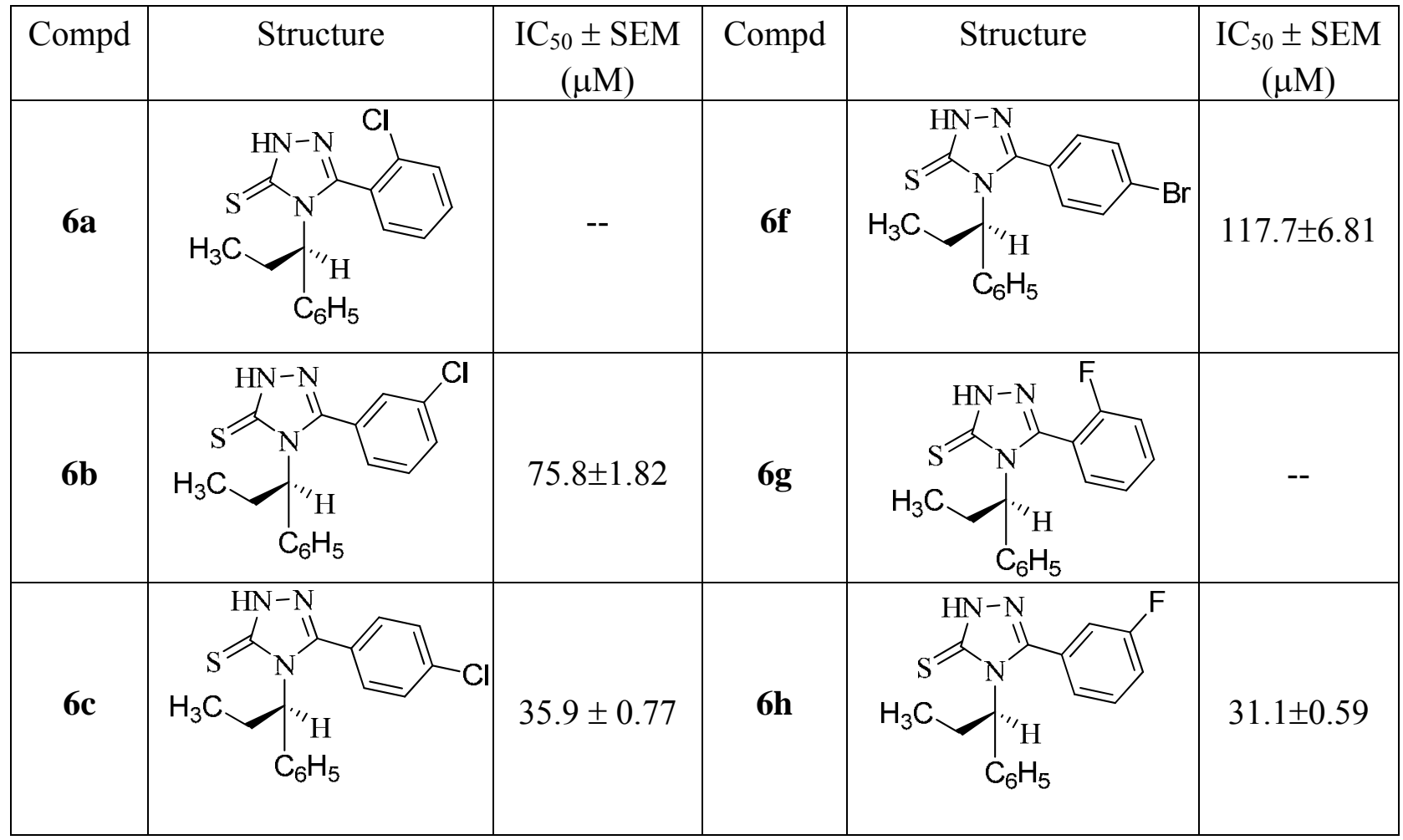




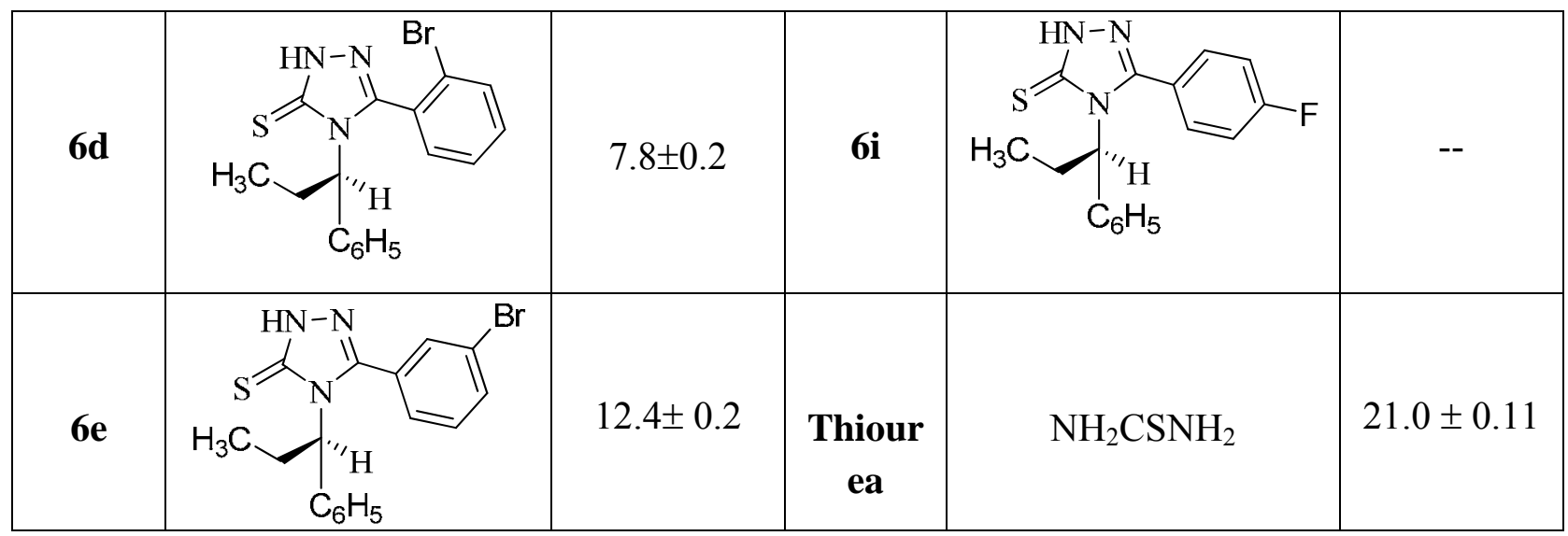

The synthesized compounds may be regarded as substrate like inhibitors on the basis of their structural similarity with the natural substrate of urease i.e., urea (Figure 2). The results revealed that the aryl part of the test compounds with its electronic effects is playing a significant role in manipulation of the activity. The most active compounds, $\mathbf{6 d}$ and $\mathbf{6 e}$, both have a bromine atom as a substituent on the aryl part, which when compared to chlorine (6d-f) and fluorine (6g-i) atoms has a lower electronegativity and donates its electrons more effectively to the phenyl ring and thus, onto the triazole nucleus. This probably positively affects the binding of the molecules to the active site of the enzyme rendering these compounds more potent than the standard thiourea.

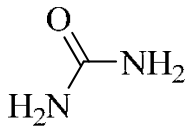

(a)

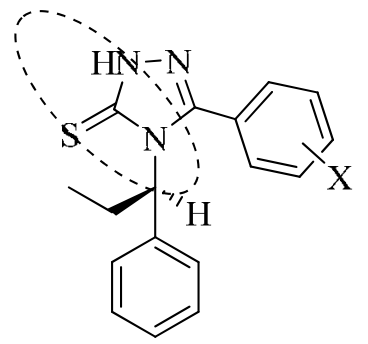

(b)

Figure 2. Representation of the possible binding to urease (a) natural substrate of urease i.e., urea (b) Substituted-1,2,4-triazole-3(4H)-thiones.

Among the fluorine substituted compounds, only the compound $\mathbf{6 h}$ exhibited very good urease inhibition $\left(\mathrm{IC}_{50}=31.1 \pm 0.5 \mu \mathrm{M}\right)$. This compound has fluorine substituent at position 3; the corresponding 2 and 4 substituted compounds were found inactive. In case of compounds having a chlorine atom as a substituent, compound $\mathbf{6 c}$ with the substituent at position 4 was found to be a very good inhibitor of urease $\left(\mathrm{IC}_{50}=35.9 \pm 0.7 \mu \mathrm{M}\right)$ whereas the compound $\mathbf{6 b}$ with the substituent at position 3 had only moderate activity $\left(\mathrm{IC}_{50}=75.8 \pm 1.8 \mu \mathrm{M}\right)$. The corresponding 2-chloro substituted compound (6a) was found to be inactive.

It may be observed from these results that among the halo-substituted compounds, a bromine atom will be the substituent of choice for good urease inhibition activity in these compounds. It 
may also be observed that the bulky chiral moiety at position 4 of the triazole nucleus has no significant effect on the urease inhibition as compounds $6 \mathbf{a}, \mathbf{6 g}$ and $6 \mathbf{i}$ were found to be inactive. The compounds $\mathbf{6 d}$ and $\mathbf{6 e}$ with a bromine substitution may serve as the lead compounds for further structural modifications. It may be suggested that the chiral moiety at position 4 of the triazole nucleus may be varied and its affect on the urease inhibition be studied. Similarly, the substitution at sulphur atom may also result in some interesting results.

\section{Antimicrobial assay}

The synthesized compounds 6a-i were tested for their anti-bacterial activity against six different bacterial strains adapting the agar well diffusion method. The bacterial strains employed were: Escherichia coli, Bacillus subtillus, Shigella flexenari, Staphylococcus aureus, Pseudomonas aeroginosa and Salmonella typhi. The anti-bacterial activity of the synthesized compounds exhibit that compounds $\mathbf{6 b}, \mathbf{6 c}$, and $\mathbf{6 e}$ have low activity against Bacillus subtillis and Staphylococcus aureus whereas compounds 6a and $6 \mathbf{i}$ have low activity against Shigella flexenari and Bacillus subtillis, respectively. Compounds $\mathbf{6 d}, \mathbf{6 f}$ and $\mathbf{6 g}$ were found to be inactive against all the bacterial strains used.

Table 2. The antibacterial activity of the compounds $\mathbf{6 a - i}$

\begin{tabular}{|c|r|r|r|r|r|c|}
\hline Test strain & $6 \mathrm{a}$ & $6 \mathrm{~b}$ & $6 \mathrm{c}$ & $6 \mathrm{e}$ & $6 \mathrm{i}$ & $\begin{array}{c}\text { Zone of } \\
\text { inhibition of Std. } \\
\text { Drug(mm) }\end{array}$ \\
\hline $\begin{array}{c}\text { Escherichia } \\
\text { coli }\end{array}$ & - & - & - & - & - & 35 \\
\hline $\begin{array}{c}\text { Bacillus } \\
\text { subtillis }\end{array}$ & - & 12 & 11 & 13 & 10 & 30 \\
\hline $\begin{array}{c}\text { Shigella } \\
\text { flexenari }\end{array}$ & 9 & 11 & 14 & - & - & 30 \\
\hline $\begin{array}{c}\text { Staphylococcus } \\
\text { aureus }\end{array}$ & - & 9 & 10 & 12 & - & 30 \\
\hline $\begin{array}{c}\text { Pseudomonas } \\
\text { aeroginosa }\end{array}$ & - & - & - & - & - & 31 \\
\hline $\begin{array}{c}\text { Salmonella } \\
\text { typhi }\end{array}$ & - & - & - & - & - & 25 \\
\hline
\end{tabular}

concentration of sample: $1 \mathrm{mg} / \mathrm{ml}$ of DMSO; size of well $6 \mathrm{~mm}$ (diameter); Std. drug: Imipenum $10 \mu \mathrm{g} /$ disc.

The compounds 6a-i were also evaluated for their in vitro antifungal activity against Trichphyton longifuses, Candida albicans, Aspergillus flavus, Microsporum canis, Fusarium 
solani and Candida glabrata, using agar tube dilution protocol. The compounds exhibited non significant activity at a sample concentration of $200 \mu \mathrm{g} / \mathrm{mL}$.

\section{Experimental Section}

General Procedures. The $\mathrm{R}_{f}$-values were determined using pre-coated silica gel aluminium packed plates, Kiesal $60 \mathrm{~F}_{254}$ from Merck (Germany). Melting points of the compounds were measured in open capillaries using Gallenkamp melting point apparatus (MP-D) and are uncorrected. The IR spectra were recorded on FTS 3000 MX, Bio-Rad Merlin (Excalibur Model) spectrophotometer. The ${ }^{1} \mathrm{H}$ - and ${ }^{13} \mathrm{C}-\mathrm{NMR}$ spectra were run on a Bruker $300 \mathrm{MHz}$ NMR spectrometer and the chemical shifts are being reported as parts per million $(\delta \mathrm{ppm})$ with the signals calibrated to the residual solvent signal. Mass spectra were recorded on Agilent technologies $6890 \mathrm{~N}$ (GC) and an inert mass selective detector 5973 mass spectrometer. The $\alpha-$ Values were measured on an Automatic Polarimeter (ATAGO AP-100). The Elemental analysis was performed on Leco CHNS-932 Elemental Analyzer, Leco Corporation (USA).

Synthesis of R-(+)-1-phenylpropyl isothiocyanate (4). R-(+)-1-Phenylpropan-1-amine (0.25 mol) was dissolved in methanol $(18 \mathrm{~mL})$ and cooled in an ice bath. Carbon disulfide $(0.38 \mathrm{~mol})$ and ammonia solution $(33 \%, 0.32 \mathrm{~mol})$ were added slowly with stirring. ${ }^{18}$ The reaction mixture was stirred for $12 \mathrm{~h}$ while maintaining the temperature around $15{ }^{\circ} \mathrm{C}$. The contents of the flask were transferred to a beaker, a solution of $\mathrm{Pb}\left(\mathrm{NO}_{3}\right)_{2}$ in water was added and stirred overnight. The isothiocyanate was collected by steam distillation. Yield 55\%; Yellow oil; $\mathrm{R}_{f}: 0.68$ (petroleum ether : ethyl acetate, $4: 1$ ); $[\alpha]_{D}^{25}-6.10^{\circ}$ (acetone C 0.0018); IR $\left(\mathrm{KBr}, \mathrm{cm}^{-1}\right) 2970$, 2943, 2084, 1601, 1454; ${ }^{1} \mathrm{H}$ NMR (300 MHz, $\left.\mathrm{CDCl}_{3}, \delta\right): 1.04$ (t, 3H, J = $\left.7.4 \mathrm{~Hz}, \mathrm{H}-3\right), 1.97$ (m, 2H, H-2), $4.72(\mathrm{dd}, 1 \mathrm{H}, J=8.1,7.4 \mathrm{~Hz}, \mathrm{H}-1), 7.23-7.44(\mathrm{~m}, 5 \mathrm{H}, \mathrm{Ar}-\mathrm{H}) ;{ }^{13} \mathrm{C}$ NMR $(75 \mathrm{MHz}$, $\left.\mathrm{CDCl}_{3}, \delta\right): 138.8,130.9,128.8,128.4,125.9,63.2,30.3,10.5$.

\section{General procedure for the synthesis of 1-aroyl-4-(1-phenylpropyl)thiosemicarbazides (5a-i)}

The substituted carboxylic acid hydrazide $(6.8 \mathrm{mmol})$ was dissolved in methanol $(30 \mathrm{~mL})$ and $\mathrm{R}-$ $(+)$-1-phenylpropyl isothiocyanate $(6.6 \mathrm{mmol})$, separately dissolved in methanol $(10 \mathrm{~mL})$, was added drop wise with continuous stirring. The reaction mixture was refluxed and monitored by TLC. After consumption of the starting materials, the reaction mixture was cooled to room temperature, concentrated in vacuo and the crude thiosemicarbazide (5a-i) recrystallized from a mixture of ethyl acetate and petroleum ether.

1-(2-Chlorobenzoyl)-4-(1-phenylpropyl)thiosemicarbazide (5a). Yield 85\%; yellow oil; $\mathrm{R}_{f}$ : 0.37 (petroleum ether : acetone; $6: 4)$; IR $\left(\mathrm{KBr}, \mathrm{cm}^{-1}\right) 3380,3169,1676,1595,1527,1231$, 1047; ${ }^{1} \mathrm{H}$ NMR $\left(300 \mathrm{MHz}\right.$, acetone- $\left.d_{6}\right) \delta 0.98(\mathrm{t}, J=7.5 \mathrm{~Hz}, 3 \mathrm{H}), 5.53(\mathrm{dd}, J=15.0,7.5 \mathrm{~Hz}, 1 \mathrm{H})$, 1.89-1.96 (m, 2H), $7.32(\mathrm{~d}, J=7.5 \mathrm{~Hz}, 2 \mathrm{H}), 7.25(\mathrm{t}, J=7.5 \mathrm{~Hz}, 1 \mathrm{H}), 7.42(\mathrm{t}, J=7.2 \mathrm{~Hz}, 2 \mathrm{H})$, 7.54-7.52 (m, 2H), 7.48-7.44 (m, 1H), $7.65(\mathrm{~d}, J=7.2 \mathrm{~Hz}, 1 \mathrm{H}), 7.66(\mathrm{~s}, 1 \mathrm{H}), 8.80(\mathrm{~s}, 1 \mathrm{H}), 9.63$ 
$(\mathrm{s}, 1 \mathrm{H}) ;{ }^{13} \mathrm{C}$ NMR $\left(75 \mathrm{MHz}\right.$, acetone- $\left.d_{6}\right) \delta 183.1,164.9,142.5,134.5,131.7,130.9,129.9,129.5$, 128.2, 127.2, 126.9, 126.8, 59.5, 28.2, 10.2; Anal. Calcd. for $\mathrm{C}_{17} \mathrm{H}_{18} \mathrm{~N}_{3} \mathrm{SOCl}$ (347.86): C, 58.70; H, 5.22; N, 12.08; S, 9.22. Found: C, 58.77; H, 5.33; N, 11.84; S, 9.27.

1-(3-Chlorobenzoyl)-4-(1-phenylpropyl)thiosemicarbazide (5b). Yield 83\%; Mp. 100-102 ${ }^{\circ} \mathrm{C}$; $\mathrm{R}_{f}: 0.35$ (petroleum ether : acetone; $\left.6: 4\right)$; IR $\left(\mathrm{KBr}, \mathrm{cm}^{-1}\right)$ 3366, 3141, 2964, 2875, 1692, 1552 , 1528, 1231, 1047; ${ }^{1} \mathrm{H}$ NMR (300 MHz, acetone- $\left.d_{6}\right) \delta 0.93(\mathrm{t}, J=7.2 \mathrm{~Hz}, 3 \mathrm{H}), 2.27-2.37(\mathrm{~m}, 2 \mathrm{H})$, $6.05(\mathrm{dd}, J=15.9,7.8 \mathrm{~Hz}, 1 \mathrm{H}), 7.44-7.48(\mathrm{~m}, 1 \mathrm{H}), 7.67(\mathrm{dt}, J=7.2,5.1 \mathrm{~Hz}, 1 \mathrm{H}), 7.72-7.77(\mathrm{~m}$, 2H), $7.85(\mathrm{dd}, J=6.9,3.3 \mathrm{~Hz}, 2 \mathrm{H}), 8.10(\mathrm{ddd}, J=7.9,1.8,1.2 \mathrm{~Hz}, 1 \mathrm{H}), 8.37(\mathrm{~d}, J=7.8 \mathrm{~Hz}$, $1 \mathrm{H}), 8.42(\mathrm{~d}, J=1.5 \mathrm{~Hz}, 1 \mathrm{H}), 8.55(\mathrm{~s}, 1 \mathrm{H}), 9.05(\mathrm{~s}, 1 \mathrm{H}), 10.29(\mathrm{~s}, 1 \mathrm{H}) ;{ }^{13} \mathrm{C} \mathrm{NMR}(75 \mathrm{MHz}$, acetone- $\left.d_{6}\right) \delta 183.9,165.4,143.3,135.0,132.3,131.6,130.8,128.5,128.0,127.3,127.2,126.6$, 60.0, 28.3, 10.2; Anal. Calcd for $\mathrm{C}_{17} \mathrm{H}_{18} \mathrm{~N}_{3} \mathrm{SOCl}$ (347.86): C, 58.70; H, 5.22; N, 12.08; S, 9.22. Found: C, 58.74; H, 5.53; N, 12.31; S, 9.30.

1-(4-Chlorobenzoyl)-4-(1-phenylpropyl)thiosemicarbazide (5c). Yield 80\%; Mp. 152-153 ${ }^{\circ} \mathrm{C}$; $\mathrm{R}_{f}: 0.37$ (petroleum ether : acetone; $\left.6: 4\right)$; IR $\left(\mathrm{KBr}, \mathrm{cm}^{-1}\right)$ 3377, 3173, 2967, 2874, 1665, 1595, 1530, 1245, 1049; ${ }^{1} \mathrm{H}$ NMR (300 MHz, acetone- $\left.d_{6}\right) \delta 0.91(\mathrm{t}, J=7.4 \mathrm{~Hz}, 3 \mathrm{H}), 1.83-1.95(\mathrm{~m}, 2 \mathrm{H})$, $5.53(\mathrm{dd}, J=15.3,7.5 \mathrm{~Hz}, 1 \mathrm{H}), 7.24-7.29(\mathrm{~m}, 5 \mathrm{H}), 7.43(\mathrm{~d}, J=8.7 \mathrm{~Hz}, 2 \mathrm{H}), 7.67(\mathrm{~s}, 1 \mathrm{H}), 7.68$ $(\mathrm{d}, J=8.7 \mathrm{~Hz}, 2 \mathrm{H}), 10.22(\mathrm{bs}, 2 \mathrm{H}) ;{ }^{13} \mathrm{C} \mathrm{NMR}(75 \mathrm{MHz}$, acetone-d 6$) \delta 178.6,161.9,139.0$, $129.4,129.3,129.1,128.6,128.3,127.5,126.6,59.7,29.2,10.6$; Anal. Calcd for $\mathrm{C}_{17} \mathrm{H}_{18} \mathrm{~N}_{3} \mathrm{SOCl}$ (347.86): C, 58.70; H, 5.22; N, 12.08; S, 9.22. Found: C, 58.75; H, 5.45; N, 11.79; S, 9.78.

1-(2-Bromobenzoyl)-4-(1-phenylpropyl)thiosemicarbazide (5d). Yield 79\%; yellow oil; $\mathrm{R}_{f}$ : 0.35 (petroleum ether : acetone; $6: 4)$; IR $\left(\mathrm{KBr}, \mathrm{cm}^{-1}\right)$ 3348, 3259, 1684, 1590, 1459, 1229, 699; ${ }^{1} \mathrm{H}$ NMR $\left(300 \mathrm{MHz}\right.$, acetone- $\left.d_{6}\right) \delta 0.96(\mathrm{t}, J=7.5 \mathrm{~Hz}, 3 \mathrm{H}), 1.99-1.93(\mathrm{~m}, 2 \mathrm{H}), 5.53(\mathrm{dd}, J=$ 15.5, 7.8 Hz, 1H), $7.26(\mathrm{dt}, J=6.9,2.7 \mathrm{~Hz}, 1 \mathrm{H}), 7.34(\mathrm{dt}, J=7.5,3.0 \mathrm{~Hz}, 2 \mathrm{H}), 7.41(\mathrm{~m}, 3 \mathrm{H})$, 7.47 (dt, $J=8.7,4.2 \mathrm{~Hz}, 1 \mathrm{H}), 7.60$ (s, 1H), 7.64 (dd, $J=7.2,1.8 \mathrm{~Hz}, 1 \mathrm{H}), 7.71$ (dd, $J=12.3,1.2$ $\mathrm{Hz}, 1 \mathrm{H}), 8.80(\mathrm{~s}, 1 \mathrm{H}), 9.61(\mathrm{~s}, 1 \mathrm{H}) ;{ }^{13} \mathrm{C} \mathrm{NMR}\left(75 \mathrm{MHz}\right.$, acetone- $\left.d_{6}\right) \delta 183.1,166.7,142.4,136.4$, 133.1, 131.8, 129.4, 129.0, 128.2, 127.7, 126.9, 126.8, 59.6, 28.2, 10.3; Anal. Calcd for $\mathrm{C}_{17} \mathrm{H}_{18} \mathrm{~N}_{3} \mathrm{SOBr}$ (392.31): C, 52.05; H, 4.62; N, 10.71; S, 8.17. Found: C, 52.20; H, 4.53; N, $10.63 ; \mathrm{S}, 8.35$.

1-(3-Bromobenzoyl)-4-(1-phenylpropyl)thiosemicarbazide (5e). Yield 84\%; Mp. $110{ }^{\circ} \mathrm{C} ; \mathrm{R}_{f}$ : 0.37 (petroleum ether : acetone; $6: 4)$; IR ( $\left.\mathrm{KBr}, \mathrm{cm}^{-1}\right)$ 3283, 3265, 2965, 2876, 1671, 1537 , 1459, 1231, 698; ${ }^{1} \mathrm{H}$ NMR (300 MHz, acetone- $\left.d_{6}\right) \delta 0.94(\mathrm{t}, J=7.5 \mathrm{~Hz}, 3 \mathrm{H}), 1.82-1.92(\mathrm{~m}, 2 \mathrm{H})$, $5.60(\mathrm{dd}, J=15.9,7.8 \mathrm{~Hz}, 1 \mathrm{H}), 7.22(\mathrm{dt}, J=6.9,2.7 \mathrm{~Hz}, 1 \mathrm{H}), 7.30(\mathrm{td}, J=7.8,3.0 \mathrm{~Hz}, 2 \mathrm{H})$, $7.39(\mathrm{dd}, J=7.8,1.8 \mathrm{~Hz}, 2 \mathrm{H}), 7.50(\mathrm{t}, J=7.9 \mathrm{~Hz}, 1 \mathrm{H}), 7.80(\mathrm{dd}, J=8.0,7.5 \mathrm{~Hz}, 1 \mathrm{H}), 7.96(\mathrm{~d}, J$ $=7.8 \mathrm{~Hz}, 1 \mathrm{H}), 8.08(\mathrm{~s}, 1 \mathrm{H}), 8.11(\mathrm{~s}, 1 \mathrm{H}), 8.59(\mathrm{~s}, 1 \mathrm{H}), 9.84(\mathrm{~s}, 1 \mathrm{H}) ;{ }^{13} \mathrm{C}$ NMR $(75 \mathrm{MHz}$, acetone$\left.d_{6}\right) \delta 183.5,164.9,142.8,134.8,134.7,131.0,130.5,128.1,126.8,126.7,126.6,121.9,59.5$, 28.2, 10.4; Anal. Calcd for $\mathrm{C}_{17} \mathrm{H}_{18} \mathrm{~N}_{3} \mathrm{SOBr}$ (392.31): C, 52.05; H, 4.62; N, 10.71; S, 8.17. Found: C, 52.17; H, 4.82; N, 10.63; S, 7.98 .

1-(4-Bromobenzoyl)-4-(1-phenylpropyl)thiosemicarbazide (5f). Yield 85\%; Mp. 160-161 ${ }^{\circ} \mathrm{C}$; $\mathrm{R}_{f}: 0.34$ (petroleum ether : acetone; $\left.6: 4\right)$; IR $\left(\mathrm{KBr}, \mathrm{cm}^{-1}\right)$ 3378, 3191, 3033, 2951, 2877, 1669, 1591, 1528, 1238, 699; ${ }^{1} \mathrm{H}$ NMR $\left(300 \mathrm{MHz}\right.$, acetone- $\left.d_{6}\right) \delta 0.97(\mathrm{t}, J=7.5 \mathrm{~Hz}, 3 \mathrm{H}), 1.82-1.92(\mathrm{~m}$, 
2H), $5.59(\mathrm{dd}, J=15.6,7.2 \mathrm{~Hz}, 1 \mathrm{H}), 7.19-7.25(\mathrm{dt}, J=7.2,3.0 \mathrm{~Hz}, 1 \mathrm{H}), 7.31(\mathrm{dt}, J=7.5,3.0$ $\mathrm{Hz}, 2 \mathrm{H}), 7.40$ (dd, $J=7.2,1.5 \mathrm{~Hz}, 2 \mathrm{H}), 7.72(\mathrm{~d}, J=8.7 \mathrm{~Hz}, 2 \mathrm{H}), 7.92(\mathrm{~d}, J=8.4 \mathrm{~Hz}, 2 \mathrm{H}), 8.06$ $(\mathrm{s}, 1 \mathrm{H}), 8.58(\mathrm{~s}, 1 \mathrm{H}), 9.81(\mathrm{~s}, 1 \mathrm{H}) ;{ }^{13} \mathrm{C} \mathrm{NMR}\left(75 \mathrm{MHz}\right.$, acetone-d $\left.d_{6}\right) \delta 183.5,165.6,142.8,133.2$, 131.6, 129.6, 128.1, 126.8, 126.7, 126.1, 59.5, 28.2, 10.4; Anal. Calcd for $\mathrm{C}_{17} \mathrm{H}_{18} \mathrm{~N}_{3} \mathrm{SOBr}$ (392.31): C, 52.05; H, 4.62; N, 10.71; S, 8.17. Found: C, 51.96; H, 4.80; N, 10.88; S, 8.07.

1-(2-Fluorobenzoyl)-4-(1-phenylpropyl)thiosemicarbazide (5g). Yield 82\%; Mp. 116-118 ${ }^{\circ} \mathrm{C}$; $\mathrm{R}_{f}: 0.35$ (petroleum ether : acetone; $\left.6: 4\right)$; IR $\left(\mathrm{KBr}, \mathrm{cm}^{-1}\right)$ 3370, 3170, 2974, 2833, 1670, 1590, 1485, 1240, 1180; ${ }^{1} \mathrm{H}$ NMR $\left(300 \mathrm{MHz}\right.$, acetone- $\left.d_{6}\right) \delta 0.90(\mathrm{t}, J=7.5 \mathrm{~Hz}, 3 \mathrm{H}), 1.79-1.85(\mathrm{~m}, 2 \mathrm{H})$, $5.40(\mathrm{dd}, J=15.5,7.3 \mathrm{~Hz}, 1 \mathrm{H}), 7.20-7.81(\mathrm{~m}, 9 \mathrm{H}), 8.08(\mathrm{~s}, 1 \mathrm{H}), 9.48(\mathrm{~s}, 1 \mathrm{H}), 10.20(\mathrm{~s}, 1 \mathrm{H}) ;{ }^{13} \mathrm{C}$ NMR $\left(75 \mathrm{MHz}\right.$, acetone- $\left.d_{6}\right) \delta 183.4,164.2,161.7(\mathrm{~d}, J=249.8 \mathrm{~Hz}), 158.3,143.4(\mathrm{~d}, J=23.0$ $\mathrm{Hz}), 133.7(\mathrm{~d}, J=8.3 \mathrm{~Hz}), 131.0(\mathrm{~d}, J=9.0 \mathrm{~Hz}), 128.7,126.9,124.9$ (d, $J=3.7 \mathrm{~Hz}), 122.2$, $116.5(\mathrm{~d}, J=22.5 \mathrm{~Hz}), 59.3,29.0,11.3$; Anal. Calcd for $\mathrm{C}_{17} \mathrm{H}_{18} \mathrm{~N}_{3} \mathrm{SOF}$ (331.12): C, 61.61; H, 5.47; N, 12.68; S, 9.68. Found: C, 61.67; H, 5.57; N, 12.75; S, 9.81.

1-(3-Fluorobenzoyl)-4-(1-phenylpropyl)thiosemicarbazide (5h). Yield 80\%; Mp. 141-142 ${ }^{\circ} \mathrm{C}$; $\mathrm{R}_{f}: 0.34$ (petroleum ether : acetone; $\left.6: 4\right)$; IR $\left(\mathrm{KBr}, \mathrm{cm}^{-1}\right) 3364,3316,2963,2873,1674,1585$, 1476, 1246, 1195; ${ }^{1} \mathrm{H}$ NMR $\left(300 \mathrm{MHz}\right.$, acetone- $\left.d_{6}\right) \delta 0.94(\mathrm{t}, J=7.5 \mathrm{~Hz}, 3 \mathrm{H}), 1.83-1.90(\mathrm{~m}, 2 \mathrm{H})$, $5.60(\mathrm{dd}, J=15.6,7.5 \mathrm{~Hz}, 1 \mathrm{H}), 7.19-7.25(\mathrm{~m}, 1 \mathrm{H}), 7.30$ (dd, $J=8.7,7.0 \mathrm{~Hz}, 2 \mathrm{H}), 7.38-7.42$ (m, $3 \mathrm{H}), 7.55-7.62(\mathrm{~m}, 1 \mathrm{H}), 7.71(\mathrm{~d}, J=9.9 \mathrm{~Hz}, 1 \mathrm{H}), 7.83(\mathrm{~d}, J=8.1 \mathrm{~Hz}, 1 \mathrm{H}), 8.05(\mathrm{~s}, 1 \mathrm{H}), 8.58(\mathrm{~s}$, $1 \mathrm{H}), 9.80(\mathrm{~s}, 1 \mathrm{H}) ;{ }^{13} \mathrm{C} \mathrm{NMR}\left(75 \mathrm{MHz}\right.$, acetone- $\left.d_{6}\right) \delta 183.5,165.0,164.1,161.0(\mathrm{~d}, J=235.5$ $\mathrm{Hz}), 142.8,130.6(\mathrm{~d}, J=7.5 \mathrm{~Hz}), 128.0,127.3,126.8(\mathrm{~d}, J=10.5 \mathrm{~Hz}), 126.5,118.8(\mathrm{~d}, J=21.0$ $\mathrm{Hz}), 114.4(\mathrm{~d}, J=23.2 \mathrm{~Hz}), 59.5,28.1,10.4$; Anal. Calcd for $\mathrm{C}_{17} \mathrm{H}_{18} \mathrm{~N}_{3} \mathrm{SOF}$ (331.12): C, 61.61; H, 5.47; N, 12.68; S, 9.68. Found: C, 61.73; H, 5.36; N, 12.73; S, 9.79.

1-(4-Fluorobenzoyl)-4-(1-phenylpropyl)thiosemicarbazide (5i). Yield 78\%; Mp. 200-202 ${ }^{\circ}$; $\mathrm{R}_{f}: 0.37$ (petroleum ether : acetone; $\left.6: 4\right)$; IR $\left(\mathrm{KBr}, \mathrm{cm}^{-1}\right) 3388,3171,3063,2971,2876,1665$, 1601, 1528, 1169; ${ }^{1} \mathrm{H}$ NMR (300 MHz, acetone- $\left.d_{6}\right) \delta 0.94(\mathrm{t}, J=7.4 \mathrm{~Hz}, 3 \mathrm{H}), 1.83-1.92(\mathrm{~m}, 2 \mathrm{H})$, $5.60(\mathrm{dd}, J=15.9,7.8 \mathrm{~Hz}, 1 \mathrm{H}), 7.19-7.24(\mathrm{~m}, 2 \mathrm{H}), 7.28-7.33(\mathrm{~m}, 3 \mathrm{H}), 7.40(\mathrm{dd}, J=8.1,1.2 \mathrm{~Hz}$, 2H), $8.05(\mathrm{dd}, J=8.7,5.4 \mathrm{~Hz}, 2 \mathrm{H}), 8.07(\mathrm{~s}, 1 \mathrm{H}), 8.55(\mathrm{~s}, 1 \mathrm{H}), 9.74(\mathrm{~s}, 1 \mathrm{H}) ;{ }^{13} \mathrm{C} \mathrm{NMR}(75 \mathrm{MHz}$, acetone- $\left.d_{6}\right) \delta 183.5,166.8,163.5(\mathrm{~d}, J=212.0 \mathrm{~Hz}), 142.9(\mathrm{~d}, J=2.8 \mathrm{~Hz}), 130.4,130.3(\mathrm{~d}, J=$ $9.0 \mathrm{~Hz}), 128.0,126.8,126.7,115.2(\mathrm{~d}, J=21.7 \mathrm{~Hz}), 59.5,28.1$, 10.4; Anal. Calcd for $\mathrm{C}_{17} \mathrm{H}_{18} \mathrm{~N}_{3} \mathrm{SOF}$ (331.12): C, 61.61; H, 5.47; N, 12.68; S, 9.68. Found: C, 61.69; H, 5.55; N, 12.80; S, 9.67 .

\section{General procedure for the synthesis of 5-aryl-4-(1-phenylpropyl)-2H-1,2,4-triazole-3(4H)-} thiones (6a-i)

The respective thiosemicarbazide $(1.4 \mathrm{mmol})$ was refluxed in $4 \mathrm{~N}$ aqueous sodium hydroxide solution $(25 \mathrm{~mL})$ and the reaction monitored by TLC. After completion, the reaction mixture was cooled to room temperature and filtered. The solid residue (if any) was discarded and the filtrate neutralized with $6 \mathrm{~N} \mathrm{HCl}$. The precipitated solid was filtered and recrystallized from aqueous ethanol. 
5-(2-Chlorophenyl)-4-(1-phenylpropyl)-2H-1,2,4-triazole-3(4H)-thione (6a). Yield 80\%; Mp. $71{ }^{\circ} \mathrm{C} ; \mathrm{R}_{f}: 0.57$ (petroleum ether : acetone; $\left.6: 4\right)$; IR (KBr, $\left.\mathrm{cm}^{-1}\right)$ 3213, 3027, 2874, 1609, 1510, 1473, 1258, 1093; ${ }^{1} \mathrm{H}$ NMR $\left(300 \mathrm{MHz}\right.$, acetone- $\left.d_{6}\right) \delta 0.99(\mathrm{t}, J=7.4 \mathrm{~Hz}, 3 \mathrm{H}), 1.89-2.13(\mathrm{~m}, 2 \mathrm{H})$, $4.66(\mathrm{dd}, J=10.6,7.5 \mathrm{~Hz}, 1 \mathrm{H}), 6.51-7.85(\mathrm{~m}, 9 \mathrm{H}), 10.55(\mathrm{~s}, 1 \mathrm{H}) ;{ }^{13} \mathrm{C}$ NMR $(75 \mathrm{MHz}$, acetone$\left.d_{6}\right) \delta 163.4,157.1,141.7,132.2,131.3,131.0,130.3,128.7,127.7,126.8,126.5,123.6,59.9$, 30.0, 10.7; MS: m/z 315, 313, 284, 198, 196, 151, 119, 113, 111, 105, 91, 77, 65; Anal. Calcd for $\mathrm{C}_{17} \mathrm{H}_{16} \mathrm{ClN}_{3} \mathrm{~S}$ (329.08): C, 61.90; H, 4.89; N, 12.74; S, 9.72. Found: C, 61.69; H, 4.55; N, 12.80; $\mathrm{S}, 9.47$.

5-(3-Chlorophenyl)-4-(1-phenylpropyl)-2H-1,2,4-triazole-3(4H)-thione (6b). Yield 82\%; yellow oil; $\mathrm{R}_{f}: 0.63$ (petroleum ether : acetone; $\left.6: 4\right)$; IR $\left(\mathrm{KBr}, \mathrm{cm}^{-1}\right) 3103,3020,2860,1614$, 1507, 1478, 1257, 1089; ${ }^{1} \mathrm{H}$ NMR $\left(300 \mathrm{MHz}\right.$, acetone- $\left.d_{6}\right) \delta 0.87(\mathrm{t}, J=7.4 \mathrm{~Hz}, 3 \mathrm{H}), 1.74-2.25$ $(\mathrm{m}, 2 \mathrm{H}), 6.29(\mathrm{dd}, J=10.5,6.0 \mathrm{~Hz}, 1 \mathrm{H}), 6.61-7.41(\mathrm{~m}, 9 \mathrm{H}), 12.5(\mathrm{~s}, 1 \mathrm{H}) ;{ }^{13} \mathrm{C} \mathrm{NMR}(75 \mathrm{MHz}$, acetone- $\left.d_{6}\right) \delta 168.5,151.0,140.3,133.4,131.2,130.5,129.8,129.3,129.2,128.1,127.2,127.1$, 58.6, 23.7, 9.6; MS: m/z 299, 297, 268, 152, 137, 119, 111, 105, 91, 77, 65; Anal. Calcd for $\mathrm{C}_{17} \mathrm{H}_{16} \mathrm{ClN}_{3} \mathrm{~S}$ (329.08): C, 61.90; H, 4.89; N, 12.74; S, 9.72. Found: C, 62.13; H, 4.97; N, 12.56; $\mathrm{S}, 9.27$.

5-(4-Chlorophenyl)-4-(1-phenylpropyl)-2H-1,2,4-triazole-3(4H)-thione (6c). Yield 81\%; Mp. $150{ }^{\circ} \mathrm{C} ; \mathrm{R}_{f}: 0.64$ (petroleum ether : acetone; $\left.6: 4\right)$; IR $\left(\mathrm{KBr}, \mathrm{cm}^{-1}\right) 3101,3038,2873,1601$, 1501, 1478, 1276, 1093; ${ }^{1} \mathrm{H}$ NMR $\left(300 \mathrm{MHz}\right.$, acetone- $\left.d_{6}\right) \delta 0.93(\mathrm{t}, J=7.4 \mathrm{~Hz}, 3 \mathrm{H}), 2.19-2.26$ $(\mathrm{m}, 2 \mathrm{H}), 6.19(\mathrm{dd}, J=10.2,6.0 \mathrm{~Hz}, 1 \mathrm{H}), 6.83-6.87(\mathrm{~m}, 2 \mathrm{H}), 7.17-7.21(\mathrm{~m}, 2 \mathrm{H}), 7.23-7.27(\mathrm{~m}$, $2 \mathrm{H}), 7.31-7.35(\mathrm{~m}, 3 \mathrm{H}), 12.2(\mathrm{~s}, 1 \mathrm{H}) ;{ }^{13} \mathrm{C}$ NMR $\left(75 \mathrm{MHz}\right.$, acetone- $\left.d_{6}\right) \delta 169.3,151.4,138.5$, $137.1,130.9$, 128.9, 128.6, 128.2, 127.1, 124.7, 59.9, 23.7, 10.7; MS: m/z 213, 211, 154, 152, 140, 138, 113, 111, 91, 75; Anal. Calcd for $\mathrm{C}_{17} \mathrm{H}_{16} \mathrm{ClN}_{3} \mathrm{~S}$ (329.08): C, 61.90; H, 4.89; N, 12.74; S, 9.72. Found: C, 61.69; H, 4.93; N, 12.39; S, 9.32 .

5-(2-Bromophenyl)-4-(1-phenylpropyl)-2H-1,2,4-triazole-3(4H)-thione (6d). Yield 75\%; Mp. $148-150^{\circ} \mathrm{C} ; \mathrm{R}_{f}: 0.64$ (petroleum ether : acetone; $\left.6: 4\right)$; IR $\left(\mathrm{KBr}, \mathrm{cm}^{-1}\right) 3087,3010,2931,1600$, 1545, 1510, 1463, 1260, 718; ${ }^{1} \mathrm{H}$ NMR (300 MHz, acetone- $\left.d_{6}\right) \delta 1.02(\mathrm{t}, J=7.4 \mathrm{~Hz}, 3 \mathrm{H}), 2.05-$ $2.28(\mathrm{~m}, 2 \mathrm{H}), 6.14(\mathrm{~d}, J=7.8 \mathrm{~Hz}, 1 \mathrm{H}), 6.19(\mathrm{dd}, J=10.8,6.3 \mathrm{~Hz}, 1 \mathrm{H}), 7.30-7.34(\mathrm{~m}, 7 \mathrm{H}), 7.65$ $(\mathrm{d}, J=8.1 \mathrm{~Hz}, 1 \mathrm{H}), 13.00(\mathrm{~s}, 1 \mathrm{H}) ;{ }^{13} \mathrm{C}$ NMR $\left(75 \mathrm{MHz}\right.$, acetone- $\left.d_{6}\right) \delta 168.5,149.7,138.1,133.1$, 132.8, 132.7, 132.3, 131.9, 128.4, 128.0, 127.8, 127.0, 59.6, 24.1, 10.9; MS: m/z 375, 373, 257 , 255, 183, 181, 118, 102, 91, 77, 65; Anal. Calcd for $\mathrm{C}_{17} \mathrm{H}_{16} \mathrm{BrN}_{3} \mathrm{~S}$ (373.02): C, 54.55; H, 4.31; N, 11.23; S, 8.57. Found: C, 54.74; H, 4.20; N, 11.81; S, 8.15.

5-(3-Bromophenyl)-4-(1-phenylpropyl)-2H-1,2,4-triazole-3(4H)-thione (6e). Yield 78\%; Mp. $61^{\circ} \mathrm{C} ; \mathrm{R}_{f}: 0.58$ (petroleum ether : acetone; $\left.6: 4\right)$; IR $\left(\mathrm{KBr}, \mathrm{cm}^{-1}\right) 3025,2961,2880,1666,1507$, 1463, 1253, 723; ${ }^{1} \mathrm{H}$ NMR (300 MHz, acetone- $\left.d_{6}\right) \delta 0.86(\mathrm{t}, J=7.4 \mathrm{~Hz}, 3 \mathrm{H}), 1.72-1.82(\mathrm{~m}, 2 \mathrm{H})$, $6.31(\mathrm{dd}, J=10.5,6.0 \mathrm{~Hz}, 1 \mathrm{H}), 6.73(\mathrm{t}, J=1.8 \mathrm{~Hz}, 1 \mathrm{H}), 6.94(\mathrm{dd}, J=8.1,0.9 \mathrm{~Hz}, 1 \mathrm{H}), 7.14-$ $7.21(\mathrm{~m}, 3 \mathrm{H}), 7.28-7.35(\mathrm{~m}, 3 \mathrm{H}), 7.52(\mathrm{ddd}, J=8.3,2.1,1.2 \mathrm{~Hz}, 1 \mathrm{H}), 10.55(\mathrm{~s}, 1 \mathrm{H}) ;{ }^{13} \mathrm{C}$ NMR $\left(75 \mathrm{MHz}\right.$, acetone- $\left.d_{6}\right) \delta 167.5,151.0,140.6,133.8,132.1,132.0,131.3,129.3,128.0,127.1$, 126.7, 121.2, 58.5, 23.8, 9.6; MS: m/z 257, 255, 198, 196, 118, 104, 91, 77, 65; Anal. Calcd for 
$\mathrm{C}_{17} \mathrm{H}_{16} \mathrm{BrN}_{3} \mathrm{~S}$ (373.02): C, 54.55; H, 4.31; N, 11.23; S, 8.57. Found: C, 54.62; H, 4.54; N, 11.07; $\mathrm{S}, 8.85$.

5-(4-Bromophenyl)-4-(1-phenylpropyl)-2H-1,2,4-triazole-3(4H)-thione (6f). Yield 76\%; Mp. $138^{\circ} \mathrm{C} ; \mathrm{R}_{f}: 0.56$ (petroleum ether : acetone; $\left.4: 6\right)$; IR (KBr, $\left.\mathrm{cm}^{-1}\right) 3104,3044,2857,1605,1501$, 1470, 1268, 1014; ${ }^{1} \mathrm{H}$ NMR $\left(300 \mathrm{MHz}\right.$, acetone- $\left.d_{6}\right) \delta 0.92(\mathrm{t}, J=7.4 \mathrm{~Hz}, 3 \mathrm{H}), 2.25-2.15(\mathrm{~m}, 2 \mathrm{H})$, 7.38-7.42 (m, 2H), 7.30-7.34 (m, 3H), 7.16-7.19 (m, 2H), $6.19(\mathrm{dd}, J=9.0,6.6 \mathrm{~Hz}, 1 \mathrm{H}), 7.55(\mathrm{~d}$, $J=8.7 \mathrm{~Hz}, 2 \mathrm{H}), 11.9(\mathrm{~s}, 1 \mathrm{H}) ;{ }^{13} \mathrm{C} \mathrm{NMR}\left(75 \mathrm{MHz}\right.$, acetone- $\left.d_{6}\right) \delta 167.2,148.9,136.0,129.0$, 128.5, 126.1, 125.6, 124.6, 122.9, 122.6, 57.4, 21.2, 8.1; MS: m/z 257, 255, 198, 196, 117, 102, 91, 77; Anal. Calcd for $\mathrm{C}_{17} \mathrm{H}_{16} \mathrm{BrN}_{3} \mathrm{~S}$ (373.02): C, 54.55; H, 4.31; N, 11.23; S, 8.57. Found: C, 54.32; H, 4.42; N, 11.49; S, 8.24.

5-(2-Fluorophenyl)-4-(1-phenylpropyl)-2H-1,2,4-triazole-3(4H)-thione (6g). Yield 73\%; Mp. $157^{\circ} \mathrm{C} ; \mathrm{R}_{f}: 0.68$ (petroleum ether : acetone; $4: 6$ ); IR $\left(\mathrm{KBr}, \mathrm{cm}^{-1}\right)$ 3086, 2960, 2878, 1625, 1510, 1474, 1258, 1193; ${ }^{1} \mathrm{H}$ NMR (300 MHz, acetone- $\left.d_{6}\right) \delta 0.98(\mathrm{t}, J=7.4 \mathrm{~Hz}, 3 \mathrm{H}), 2.05-2.32$ $(\mathrm{m}, 2 \mathrm{H}), 6.14(\mathrm{dd}, J=9.9,6.6 \mathrm{~Hz}, 1 \mathrm{H}), 6.14-7.60(\mathrm{~m}, 9 \mathrm{H}), 13.01(\mathrm{~s}, 1 \mathrm{H}) ;{ }^{13} \mathrm{C} \mathrm{NMR}(75 \mathrm{MHz}$, acetone- $\left.d_{6}\right) \delta 169.9,160.6(\mathrm{~d}, J=247.5 \mathrm{~Hz}), 158.9,138.1,133.1(\mathrm{~d}, J=8.3 \mathrm{~Hz}), 132.2(\mathrm{~d}, J=$ $1.5 \mathrm{~Hz}), 128.2,127.8,127.3,124.0(\mathrm{~d}, J=3.7 \mathrm{~Hz}), 115.6(\mathrm{~d}, J=21.0 \mathrm{~Hz}), 114.9(\mathrm{~d}, J=15.0$ $\mathrm{Hz})$, 59.2, 23.7, 10.2; MS: $\mathrm{m} / \mathrm{z}$ 313, 195, 136, 118, 104, 91, 77; Anal. Calcd for $\mathrm{C}_{17} \mathrm{H}_{16} \mathrm{FN}_{3} \mathrm{~S}$ (313.1): C, 65.15; H, 5.15; N, 13.41; S, 10.23. Found: C, 65.39; H, 4.93; N, 13.81; S, 9.87.

5-(3-Fluorophenyl)-4-(1-phenylpropyl)-2H-1,2,4-triazole-3(4H)-thione (6h). Yield 75\%; yellow oil; $\mathrm{R}_{f}$ : 0.61 (petroleum ether : acetone; $\left.6: 4\right)$; IR $\left(\mathrm{KBr}, \mathrm{cm}^{-1}\right) 3092,3021,2925,1610$, 1513, 1470, 1261, 1184; ${ }^{1} \mathrm{H}$ NMR $\left(300 \mathrm{MHz}\right.$, acetone- $\left.d_{6}\right) \delta 0.97(\mathrm{t}, J=7.3 \mathrm{~Hz}, 3 \mathrm{H}), 2.18-2.25$ $(\mathrm{m}, 2 \mathrm{H}), 6.21(\mathrm{dd}, J=10.5,6.0 \mathrm{~Hz}, 1 \mathrm{H}), 6.57-7.43(\mathrm{~m}, 9 \mathrm{H}), 12.1(\mathrm{~s}, 1 \mathrm{H}) ;{ }^{13} \mathrm{C} \mathrm{NMR}(75 \mathrm{MHz}$, acetone- $\left.d_{6}\right) \delta 169.2,161.5(\mathrm{~d}, J=241.0 \mathrm{~Hz}), 151.1,138.4,130.0(\mathrm{~d}, J=8.3 \mathrm{~Hz}), 128.6,128.1(\mathrm{~d}$, $J=9.7 \mathrm{~Hz}), 127.1,126.5,125.5(\mathrm{~d}, J=3.0 \mathrm{~Hz}), 117.8(\mathrm{~d}, J=21.0 \mathrm{~Hz}), 116.9(\mathrm{~d}, J=23.3 \mathrm{~Hz})$, 59.9, 23.8, 10.7; MS: $m / z$ 313, 195, 136, 119, 104, 91, 77, 65; Anal. Calcd for $\mathrm{C}_{17} \mathrm{H}_{16} \mathrm{FN}_{3} \mathrm{~S}$ (313.1): C, 65.15; H, 5.15; N, 13.41; S, 10.23. Found: C, 65.55; H, 5.21; N, 13.21; S, 9.98.

5-(4-Fluorophenyl)-4-(1-phenylpropyl)-2H-1,2,4-triazole-3(4H)-thione (6i). Yield 77\%: Mp. $143^{\circ} \mathrm{C} ; \mathrm{R}_{f}: 0.71$ (petroleum ether : acetone; $\left.6: 4\right)$; IR $\left(\mathrm{KBr}, \mathrm{cm}^{-1}\right)$ 3096, 3054, 2930, 1607, 1514, 1480, 1292, 1163; ${ }^{1} \mathrm{H}$ NMR (300 MHz, acetone- $\left.d_{6}\right) \delta 0.94(\mathrm{t}, J=7.4 \mathrm{~Hz}, 3 \mathrm{H}), 2.24-1.88(\mathrm{~m}, 2 \mathrm{H})$, $6.19(\mathrm{dd}, J=10.8,6.3 \mathrm{~Hz}, 1 \mathrm{H}), 6.86-6.97$ (m, 4H), 7.16-7.19 (m, 2H), 7.34-7.31 (m, 3H), 11.8 $(\mathrm{s}, 1 \mathrm{H}) ;{ }^{13} \mathrm{C} \mathrm{NMR}\left(75 \mathrm{MHz}\right.$, acetone- $\left.d_{6}\right) \delta 172.6,162.3(\mathrm{~d}, J=236.5 \mathrm{~Hz}), 153.2,138.5(\mathrm{~d}, J=$ $3.1 \mathrm{~Hz}), 131.8$ (d, $J=24.1 \mathrm{~Hz}), 128.6,128.1,127.1,122.3,115.5$ (d, $J=12.3 \mathrm{~Hz}), 59.8,23.7$, 10.7; MS: $\mathrm{m} / \mathrm{z}$ 313, 195, 136, 119, 104, 91, 77, 65; Anal. Calcd for $\mathrm{C}_{17} \mathrm{H}_{16} \mathrm{FN}_{3} \mathrm{~S}$ (313.1): C, 65.15; H, 5.15; N, 13.41; S, 10.23. Found: C, 65.51; H, 5.27; N, 13.29; S, 10.01.

\section{Urease assay and inhibition ${ }^{14}$}

A solution comprising $25 \mu \mathrm{L}$ of Jack bean Urease, $55 \mu \mathrm{L}$ of buffer and $100 \mathrm{mM}$ urea were incubated with $5 \mu \mathrm{L}\left(0.5 \mathrm{mM}\right.$ conc.) of the test compounds at $30{ }^{\circ} \mathrm{C}$ for $15 \mathrm{~min}$ in well plates. The production of ammonia was measured by indophenol method and used to determine the urease inhibitory activity. The phenol reagent $(45 \mu \mathrm{L}, 1 \% \mathrm{w} / \mathrm{v}$ phenol and $0.005 \% \mathrm{w} / \mathrm{v}$ sodium 
nitroprusside) and alkali reagent $(70 \mu \mathrm{L}, 0.5 \% \mathrm{w} / \mathrm{v}$ sodium hydroxide and $0.1 \% \mathrm{NaOCl})$ were added to each well and the increasing absorbance at $630 \mathrm{~nm}$ was measured after $50 \mathrm{~min}$, using a microplate reader (Molecular Device, USA). The change in absorbance per min was noted and the results processed using SoftMax Pro software (Molecular Device, USA). All the tests were performed in triplicate. The assays were performed at $\mathrm{pH} 8.2\left(0.01 \mathrm{M} \mathrm{K}_{2} \mathrm{HPO}_{4} .3 \mathrm{H}_{2} \mathrm{O}, 1.0 \mathrm{mM}\right.$ EDTA and $0.01 \mathrm{M} \mathrm{LiCl}_{2}$ ). The percentage inhibition was calculated from the formula $100-$ $\left(\mathrm{OD}_{\text {testwell }} / \mathrm{OD}_{\text {control }}\right) \times 100$. Thiourea was used as the standard inhibitor.

\section{Acknowledgements}

The authors are thankful to Higher Education Commission (HEC) of Pakistan for the financial support through project No. 20-674/R\&D/06/1764 under National Research Program for Universities.

\section{References}

1. Al-Masoudi, I. A.; Al-Soud, Y. A.; Al-Salihi, N. J.; Al-Masoudi, N. A. Chem. Heterocycl. Compds. 2006, 42, 1377.

2. Torres, H. A.; Hachem, R. Y.; Chemaly, R. F.; Kontoyiannis, D. P.; Raad, I. I. Lancet Infect. Dis. 2005, 5, 775.

3. Chai, B.; Qian, X.; Cao, S.; Liu, H.; Song, G. Arkivoc 2003, (ii), 141.

4. $\quad$ El-masry, A. H.; Fahmy, H. H.; Abdelwahed, S. H. A. Molecules 2000, 5, 1429.

5. Orabi, A. S.; Moneim, M. A.; El-Din salem, E.; El-Din, M. A. -F. Polish J. Chem. 2000, $74,1675$.

6. Witkowski, J. J.; Robin, R. K.; Sidwell, R. W.; Simon, L. N. J. Med. Chem. 1972, 15, 1150.

7. Emami, S.; Falahati, M.; Banifatemi, A.; Amanlou, M.; Shafiee, A. Bioorg. Med. Chem. 2004, 12, 3971.

8. Kucukguzel, I.; Tatar, E.; Kucukguzel, G. S.; Rollas, S.; De Clercq, E. Eur. J. Med. Chem. 2008, 43, 381.

9. George, T.; Mehta, D. V.; Tahilramani, R.; David, J.; Talwalker, P. K. J. Med. Chem. 1971, 14, 335 .

10. Bentiss, F.; Lagrenee, M.; Traisnel, M.; Mernari, B.; Elattari, H. J. Appl. Electrochem. 1999, 29, 1073.

11. Naud, D. L.; Hiskey, M. A.; Harry, H. H. J. Energ. Mater. 2003, 21, 57.

12. Akhtar, T.; Hameed, S.; Al-Masoudi, N. A.; Loddo, R.; La Colla, P. Acta Pharm. 2008, $58,135$. 
13. Akhtar, T.; Hameed, S.; Al-Masoudi, N. A.; Khan, K. M. Heteroatom Chem. 2007, 18, 316.

14. Akhtar, T.; Hameed, S.; Khan, K. M.; Chaudhary, M. I. Med. Chem. 2008, 4, 539.

15. Akhtar, T.; Hameed, S.; Zia-ur-Rehman, M.; Bukhari, T. H.; Khan, I. Acta Cryst. 2008, E64, o1388.

16. Akhtar, T.; Hameed, S.; Lu, X.; Yasin, K. A.; Khan, M. H. Analytical Sciences: X-ray Structure Analysis Online 2006, 22, 307.

17. Furniss, B. S.; Hannaford, A. J.; Smith, P. W. G.; Tatchell, A. R. Vogel's Text Book of Practical Organic Chemistry, $5^{\text {th }}$ Edn.; Longman Scientific and Technical, copublished in the United States with John Wiley and Sons Inc.: New York, 1991; pp 1077 \& 1269.

18. Zamani, K.; Faghihi, K.; Sangi, M. R., Zolgharnein, J. Turk. J. Chem. 2003, 27, 119. 